\title{
SCP special issue on Bytecode 2012 - Preface
}

Bytecode, such as produced by, e.g., Java and .NET compilers, has become an important topic of interest, both for industry and academia. The industrial interest stems from the fact that bytecode is typically used for Internet and mobile device applications (smart cards, phones, etc.), where security is a major issue. Moreover, bytecode is device-independent and allows dynamic loading of classes, which provides an extra challenge for the application of formal methods. Also the unstructuredness of the code and the pervasive presence of the operand stack provide further challenges for the analysis of bytecode. Therefore, there is a high demand to study the theoretical and practical aspects of semantics, verification, analysis, certification and transformation of bytecode.

Research on bytecode is an ideal test bench for the application of formal methods to real languages. There is a major pressure in this sense, because security is a hot topic for bytecode applications in embedded devices. However, the scientific community on bytecode semantics, verification, analysis, and transformation is currently fragmented, and researchers often come from the two distinct worlds of industry and academia.

To create a bridge between these two distinct worlds, a series of workshops on all aspects of bytecode semantics, verification, analysis and transformation has been organised as a satellite event of ETAPS. In 2012, the 7th edition of this Bytecode workshop was held as a satellite of ETAPS 2012 in Tallinn, Estonia. During the workshop, researchers and practitioners from both the industrial and the academic world presented new results and demonstrated new software tools that are of interest for the community as a whole.

This special issue presents a selection of papers that originate from this workshop. After the workshop, the best submitted papers were invited to submit an extended version to this special issue. Additionally, also the workshop's invited speakers were invited to contribute to the special issue. The papers underwent a thorough reviewing process, with several iterations. In addition to the original PC members, all papers were also reviewed by external domain experts. As a result of this process, three interesting papers presenting different aspects of semantics, verification, analysis, and transformation of bytecode are published in this SCP special issue.

Elvira Albert, Puri Arenas, Samir Genaim, Germán Puebla, and Guillermo Román-Díez write about Conditional Termination of Loops over Heap-Allocated Data. In this paper they describe a novel approach for termination analysis of Java bytecode programs, where termination is heap-sensitive, i.e., it depends on data. They do this by defining an equivalence-preserving transformation that finds fields that can be replaced by new "ghost variables", which allows then to reuse existing heapinsensitive termination analysis techniques.

Eric R. Wognsen, Henrik S. Karlsen, Mads C. Olesen, and René R. Hansen describe Formalisation and Analysis of Dalvik Bytecode. They provide the first formalisation of the complete Dalvik bytecode language including reflection features and the first formally-specified control flow analysis for the language, including advanced control flow features such as dynamic dispatch, exceptions, and reflection.

Finally, Víctor Braberman, Diego Garbervetsky, Samuel Hym, and Sergio Yovine present Summary-Based Inference of Quantitative Bounds of Live Heap Objects, a symbolic static analysis using method summaries for computing parametric upper bounds of the number of simultaneously live objects of sequential Java-like programs.

Guest Editor Marieke Huisman Formal Methods and Tools Group University of Twente Netherlands 